\title{
DAT Genotype Modulates Brain and Behavioral Responses Elicited by Cigarette Cues
}

\author{
Teresa R Franklin*,', Falk W Lohoff', Ze Wang', Nathan Sciortino', Derek Harper', Yin Li', Will Jens', \\ Jeffrey Cruz', Kyle Kampman ${ }^{1,2}$, Ron Ehrman ${ }^{1,2}$, Wade Berrettini', John A Detre ${ }^{3,4}$, Charles P O'Brien ${ }^{1,2}$ \\ and Anna Rose Childress ${ }^{1,2}$
}

'Department of Psychiatry, Addiction Treatment Research Center, University of Pennsylvania, Philadelphia, PA, USA; ${ }^{2}$ VA VISN 4 MIRECC, Philadelphia, Department of Veteran's Affairs Medical Center, Philadelphia, PA, USA; ${ }^{3}$ Department of Radiology, University of Pennsylvania, Philadelphia, PA, USA; ${ }^{4}$ Department of Neurology, University of Pennsylvania, Philadelphia, PA, USA

\begin{abstract}
We previously demonstrated differential activation of the mesocorticolimbic reward circuitry in response to cigarette cues independent of withdrawal. Despite robust effects, we noted considerable individual variability in brain and subjective responses. As dopamine (DA) is critical for reward and its predictive signals, genetically driven variation in DA transmission may account for the observed differences. Evidence suggests that a variable number of tandem repeats (VNTRs) polymorphism in the DA transporter (DAT) SLC6A3 gene may influence DA transport. Brain and behavioral responses may be enhanced in probands carrying the 9-repeat allele. To test this hypothesis, perfusion $\mathrm{MR}$ images were acquired during cue exposure in 19 smokers genotyped for the 40 bp VNTR polymorphism in the SLC6A3 gene. Contrasts between groups revealed that 9-repeat (9-repeats) had a greater response to smoking (vs nonsmoking) cues than smokers homozygous for the 10-repeat allele (10/10-repeats) bilaterally in the interconnected ventral striatal/pallidal/orbitofrontal cortex regions (VS/VP/OFC). Activity was increased in 9-repeats and decreased in 10/I0-repeats in the VS/VP/OFC ( $p<0.00 \mathrm{I}$ for all analyses). Brain activity and craving was strongly correlated in 10/10-repeats in these regions and others (anterior cingulate, parahippocampal gyrus, and insula; $r^{2}=0.79-0.86, p<0.00$ I in all regions). Alternatively, there were no significant correlations between brain and behavior in 9-repeats. There were no differences in cigarette dependence, demographics, or resting baseline neural activity between groups. These results provide evidence that genetic variation in the DAT gene contributes to the neural and behavioral responses elicited by smoking cues.

Neuropsychopharmacology (2009) 34, 717-728; doi:I0.1038/npp.2008. I24; published online I3 August 2008
\end{abstract}

Keywords: $\mathrm{fMRI}$; dopamine transporter; gene; smoking; reward; cue

\section{INTRODUCTION}

Cigarette smoking persists as the premier cause of preventable death in the United States and is the direct cause of most lung cancer, chronic obstructive pulmonary disease, emphysema, and numerous other devastating and fatal diseases (Centers for Disease Control and Prevention, 1993; Cancer Facts and Figures, 2007). In total, $40 \%$ of the approximately 36 million smokers in the United States attempt to quit each year however less than $7 \%$ succeed, even when assisted with nicotine replacement (Baillie et al, 1995; Hughes et al, 2003). During abstinence, smokers are often obsessively fixated on smoking to alleviate intolerable cravings, induced by nicotine withdrawal and/or reminders

*Correspondence: Dr TR Franklin, Department of Psychiatry, Addiction Treatment Research Center, University of Pennsylvania and Philadelphia VA Medical Center, 3900 Chestnut Street, Philadelphia, PA 19104, USA, Tel: + I 2152223200 , ext. I19, Fax: + I 215386 6770, E-mail: franklin_t@mail.trc.upenn.edu

Received 16 April 2008; revised II July 2008; accepted I 5 July 2008 to smoke, both of which contribute to relapse (Baker et al, 1986; Shiffman et al, 1996). Nicotine withdrawal symptoms abate within 1 month of quitting (Hughes, 2007). However, smokers report that reminders to smoke such as the smell of a burning match, another person smoking, and even internal mood states repeatedly associated with smoking can trigger relapse months or even years after quitting.

A wealth of preclinical literature examining the neurobiology underlying drug dependence and the cues that predict their availability point to a final common brain substrate, the mesolimbic dopaminergic reward system (Di Chiara and Imperato, 1988; Di Ciano et al, 1998; Duvauchelle et al, 2000; Gratton and Wise, 1994). Current neuroimaging techniques provide the opportunity to examine dopaminergic systems in humans. Volkow et al (2006), followed by Wong et al (2006) were the first to provide direct evidence that cocaine cues on their own elicit craving and increased dopamine (DA) release in the striatum of cocaine-addicted individuals. This work was followed by the study of Boileau et al (2007) who showed 
that only three pairings with amphetamine produced conditioned DA release in the ventral striatum (VS) of normal volunteers accompanied by amphetamine-like behavioral responses.

We showed robust and consistent brain perfusion, independent of withdrawal, to smoking cues (SCs) in reward-related mesocorticolimbic circuitry (amygdala, VS, orbitofrontal cortex, parahippocampus, medial thalamus, and insula) that correlated with craving in posterior cingulate and dorsolateral prefrontal cortex (Franklin et al, 2007) supporting hypotheses implicating this circuitry in stimulus-evoked craving and relapse (Corrigall et al, 1992; Di Chiara, 2000; Franklin and Druhan, 2000a,b). Despite a robust overall cue effect, there was considerable interindividual variability in the brain response. As heritability estimates for various smoking behaviors range from 46 to $84 \%$ (Batra et al, 2003; Heath and Madden, 1995), and as DA is a critical neurotransmitter for drug reward and is released by its signals, we hypothesized that genetically driven variation in DA transmission contributes to differences in the strength of the attribution of incentive salience to cues associated with smoking cigarettes and may be reflected in different neural activation patterns and/or intensity of activations.

In the present studies, we linked functional neuroimaging and behavioral measurements of subjective craving with a candidate gene approach to evaluate the impact of genetic variation in the dopamine transporter (DAT) gene (SLC6A3) on brain perfusion to SCs. The DAT is abundantly present in the VS and rapidly clears DA from the synapse after phasic release that occurs in response to primary rewards and predictive stimuli (Jaber et al, 1997; Mash et al, 2002; Zahniser and Sorkin, 2004). Evidence suggests that a $40 \mathrm{bp}$ variable number of tandem repeat (VNTR) polymorphism in the $3^{\prime}$-untranslated region of the SLC6A3 effects DAT expression (Vandenbergh et al, 1992). The 9repeat allele (9-repeats) is associated with lower DAT expression (Fuke et al, 2001; Mill et al, 2002) (although see van Dyck et al, 2005), which may result in slow DA clearance potentially reflecting prolongation of the DArelated 'reward message'. In support of this view, Erblich et al (2005) found that smokers carrying a 9-repeat reported stronger SC-induced cravings. Further support is provided by a study in which ${ }^{11} \mathrm{C}$ raclopride competition in striatal regions was greater in 9-repeats immediately after smoking (Brody et al, 2006), which might indicate either compromised DAT function or reduced availability in the 9-repeat probands.

These and other studies provide the foundation for our hypothesis that synaptic DA levels are sustained in smokers with the less 'active' or less available form of the DAT and may be reflected in a heightened neural response to SCs. Particularly, as in our previous study, we expected carriers of a 9-repeat to exhibit increased activity in the VS, orbitofrontal cortex (OFC), amygdala, parahippocampal gyrus, thalamus and insula, and would report greater craving to the SCs compared to probands homozygous for the 10-repeat allele (10/10-repeats). To test this hypothesis, we utilized the technique of continuous arterial spin-labeled (CASL) perfusion fMRI, which is quantitative and stable over time and thus well suited for the study of low frequency (sustained) brain changes (Detre et al, 1992), such as drug craving, which once triggered, can persist for several minutes (Childress et al, 1999; Franklin et al, 2007; Volkow et al, 2006).

\section{Subjects}

The study, approved by the University of Pennsylvania Institutional Review Board, adhered to the Declaration of Helsinki. Smokers were compensated $\$ 100$ for completion of both MRI scans. Subjects $(\sim 75 \%)$ were recruited from those presenting for treatment for nicotine dependence at the University of Pennsylvania Treatment Research Center and through word of mouth $(\sim 25 \%)$. Subjects were screened, tested on study knowledge, and consented prior to psychological and physical evaluations. The Minnesota International Neuropsychiatric Interview (Sheehan et al, 1998) is a short accurate structured diagnostic psychiatric interview, which was used to determine current DSM-IV diagnosis of psychoactive substance dependence other than nicotine and to diagnose current severe psychiatric symptoms. Individuals with other current psychoactive substance dependence or current DSM-IV psychiatric diagnoses were excluded. Severity of nicotine dependence was determined from a laboratory-developed Smoking History Questionnaire that included a modified Fagerstrom Test for Nicotine Dependence (FTND; Fagerstrom and Schneider, 1989).

Individuals with an abnormal structural MRI, a history of head trauma or injury causing loss of consciousness lasting greater than $3 \mathrm{~min}$ or associated with skull fracture or intercranial bleeding, or had magnetically active objects on or within their body were excluded.

DNA samples were acquired and functional scanning was performed on 22 subjects, 3 of whom were excluded due to incomplete image acquisition. A subgroup of these subject's imaging data has been published previously (Franklin $e t a l$, 2007). Thus, the final sample consisted of $19(N=10 \mathrm{men}$, $32 \%$ African American, 43\% European American, 21\% multiple ethnicity) physically healthy and mentally stable smokers between the ages of 18 and $60(35.8 \pm 2.38)$ who met DSM-IV criteria for nicotine dependence (FTND: 4.77 $( \pm 0.52)$; indicating moderate dependence). Subjects smoked from 12 to 35 cigarettes per day $(21.4 \pm 1.61)$ and averaged $13.3( \pm 0.47)$ years of education.

\section{METHODS}

\section{Imaging Approach}

Continuous arterial spin-labeled perfusion fMRI, an indirect measurement of neural activity (Floyd et al, 2003), was used to test whether brain and behavioral responses to SCs would be heightened in probands carrying an SLC6A3 9-repeat (9-repeats) vs probands homozygous for the 10/10-repeat (10-10 repeats). This technique has been previously described in detail (Detre et al, 1992; Detre and Wang, 2002). Perfusion fMRI provides a quantitative measurement of cerebral blood flow (CBF; ml of blood per $100 \mathrm{~g}$ of tissue per minute) permitting comparisons of changes in regional activity across multiple scanning sessions. This feature is beneficial for the study of longitudinal or very lowfrequency brain changes, such as the drug craving state. 
Thus, it is particularly useful in our SC paradigm wherein smokers enter and exit the scanner between cue sets to smoke a cigarette.

\section{Scanning Procedures and Stimulus Description}

The cue-reactivity procedure exposes subjects to a variety of smoking-related stimuli whereas subjective reports of craving and functional MRI data are acquired.

Two separate scanning sessions (counterbalanced with respect to SC and non-SCs) were administered, $1 \mathrm{~h}$ apart and preceded by smoking to minimize 'carry over' craving and pharmacologic withdrawal that can accrue throughout scanning. For each session, a $10 \mathrm{~min}$ CASL scan was acquired during cue exposure approximately 20-25 min after smoking to ensure dissipation of the acute cardiovascular effects of smoking. Subjects were informed of the opportunity to smoke after each scan as expectancy enhances subjective craving and physiological responses (Droungas et al, 1995; McBride et al, 2006). Craving and withdrawal reports were obtained before and after each scan using the Craving and Withdrawal Questionnaire (Table 1).

Cue exposure consisted of two 10-min stimulus sets: a non-SC audiovisual clip comprised of several individuals (differing in race, age, and sex) relating interesting short stories or anecdotes, or a SC video containing similar footage but featuring smoking, cigarette cues, and explicit language designed to induce appetitive desire for a cigarette. Smokers held one of their own cigarettes during the SC scan and a pencil during the non-SC scan. Cue sets have been previously validated in Franklin et al (2007).

\section{Imaging Parameters and Data Processing}

MR scanning parameters were previously described in detail in Franklin et al (2007). Briefly, data was acquired on a $3.0 \mathrm{~T}$ Trio whole-body scanner (Siemens AG, Erlangen, Germany), using a standard volume coil. A T1-weighted threedimensional HR MPRAGE scan was acquired $(\mathrm{FOV}=250 \mathrm{~mm}, \mathrm{TR} / \mathrm{TE}=1620 / 3 \mathrm{~ms}, 192 \times 256$ matrix, slice thickness $1 \mathrm{~mm}$ ) for anatomical coregistration and spatial normalization. CASL perfusion fMRI was used to acquire the resting baseline (5 min) and SC and non-SC (10 min) scans. Interleaved images with and without labeling were obtained using a gradient echo and echo-planar-imaging

Table I Table of Demographics (Means \pm SEMs)

\begin{tabular}{lcc}
\hline Demographic & 9-repeats & I O/I O-repeats \\
\hline Age & $37.9( \pm 4.1)$ & $33.8( \pm 2.8)$ \\
Education & $13.89( \pm 0.68)$ & $12.69( \pm 0.64)$ \\
Sex & Male $(6)$, Female $(4)$ & Male (4), Female (5) \\
Pack years & $22.88( \pm 5.5 \mathrm{I})$ & $20.92( \pm 6.04)$ \\
Cigarettes per day & $19.89( \pm 2.64)$ & $23.13( \pm 1.42)$ \\
FTND scores & $4.29( \pm 0.77)$ & $5.19( \pm 0.89)$ \\
Race & Black (4), White $(4)$ & Black (3), White (4) \\
& Multiple (2) & Multiple $(2)$ \\
\hline
\end{tabular}

There were no significant differences in any of the above characteristics between groups. sequence with a delay of $700 \mathrm{~ms}$ inserted between the end of the labeling pulse and image acquisition $(\mathrm{FOV}=220 \mathrm{~mm}$, matrix $=64 \times 64 \times 14, \mathrm{TR} / \mathrm{TE}=3000 / 17 \mathrm{~ms}$, flip angle $=90^{\circ}$, slice thickness $=8 \mathrm{~mm}$ with a $2 \mathrm{~mm}$ interslice gap.

\section{Imaging Data Processing and Statistical Analyses}

An SPM2-based (Wellcome Department of Cognitive Neurology, London, UK) ASL data processing toolbox (Wang et al, 2008) was used for data analyses as described previously (Franklin et al, 2007). Briefly, ASL image pairs were realigned to the mean of all control images and spatially smoothed with a three-dimensional isotropic Gaussian kernel (FWHM, $10 \mathrm{~mm}$ ). CBF image series were generated using a simplified two-compartment model with the simple-subtraction method for $\mathrm{CBF}$ calculations (Aguirre et al, 2005). The mean control image of each subject's data was coregistered to the structural image using the mutual information based coregistration algorithm provided by SPM2. The same coregistration parameters were also used to coregister the CBF maps to the structural image. The structural image was then spatially normalized to the Montreal Neurological Institute (MNI) standard brain. The same parameters were used to normalize the CBF images to the MNI standard space. Each subject's normalized mean control images were segmented using SPM2. The segmented gray matter masks were averaged and the overlap of subject's gray matter was extracted. This final mask was used for calculating global CBF for each session.

\section{Statistical Analyses}

Voxel-wise analyses of the CBF data were conducted on each subject, using a general linear model (GLM) with global CBF time course and age as nuisance covariates added into the model. There was no temporal filtering or smoothing. All preprocessing was done blinded and prior to subsequent group assignment. Contrasts between cue sets were defined in the GLM model to assess the voxel-by-voxel CBF difference. Using the corresponding parametric maps of this contrast (the $\beta$ maps), random effects analysis was employed to test for a significant main effect of condition (SC vs non-SC) in each genotype with a statistical parametric map of the $T$-statistic at each voxel for population inference (second-level analysis). This two stage analysis is theoretically equivalent to a two way ANOVA (Penny et al, 2003).

Simple regression analyses were conducted on CBF with the change in craving scores as the covariate of interest (post- (-) pre-SC craving scores)) to test for brain/ behavioral correlations.

\section{Genotyping}

Genomic DNA was extracted from anti-coagulated venous blood samples using a standard salting out method (Lahiri and Nurnberger, 1991). Genotyping of the SLC6A3 $40 \mathrm{bp}$ repeat polymorphism (rs28363170) was performed as previously described (Vandenbergh et al, 1992). Briefly, PCR was performed using a mix of $100 \mathrm{ng}$ genomic DNA, $1 \times$ AmpliTaq buffer containing $\mathrm{MgCl}_{2}, 200 \mathrm{~nm}$ dNTP mix, $150 \mathrm{nmol}$ for each primer (forward: $5^{\prime}$-TGTGGTGTAGG 
GAACGGCCTGAG-3' and reverse: $5^{\prime}$-CTTCCTGGAGGTC ACGGCTCAAGG-3' ${ }^{\prime}$ ) and $2.5 \mathrm{U}$ AmpliTaq per reaction. The PCR conditions included an initial melting step $\left(94^{\circ} \mathrm{C}\right.$; $4 \mathrm{~min}$ ) followed by 40 cycles of melting $\left(94^{\circ} \mathrm{C} ; 1 \mathrm{~min}\right)$, annealing $\left(65^{\circ} \mathrm{C} ; 1 \mathrm{~min}\right)$, and extending $\left(72^{\circ} \mathrm{C} ; 1 \mathrm{~min}\right)$. A final extension step was used $\left(72^{\circ} \mathrm{C} ; 5 \mathrm{~min}\right)$. Reaction products were separated by agarose gel electrophoresis, and product sizes were determined by comparison to molecular weight standards and known sequenced samples. In addition, four samples were confirmed by sequencing. All samples were run in duplicate with $100 \%$ concordance rate and read independently by two blinded investigators.

\section{RESULTS}

\section{Group Assignment}

Nineteen smokers were genotyped for variance in the SLC6A3. Of which, 9 subjects were homozygous for the 10/ 10 -repeats whereas 8 were heterozygous and 2 homozygous for the 9-repeat. Genotype frequencies did not deviate from that expected under Hardy-Weinberg equilibrium $\left(\chi^{2}=0.1067\right)$. As noted previously, smokers who carried at least one 9-repeat were classified and grouped together for analyses as 9-repeats and 10/10-repeat probands were classified as 10/10-repeats resulting in allele frequencies of 0.32 for 9-repeats and 0.68 for 10/10-repeats, which is similar to that found in other studies (Vandenbergh et al, 2002). Both groups contained similar numbers of treatment seekers $v s$ nontreatment seekers.

\section{Demographics}

As shown in Table 1, there were no significant differences between genotypes in ethnicity, sex, cigarettes smoked per day, pack years (a measure to quantify intensity of chronic cigarette exposure since smoking initiation) or other general demographic items.

\section{Subjective Measures}

Subjects rated their craving, withdrawal, mood state, and interest in the video before and after stimulus presentations using a 9-item Craving and Withdrawal Questionnaire. For each item, reported scores are the post- $(-)$ pre-stimulus scores. All items were analyzed using paired $t$-tests except for item 3, intended to assess craving, which we predicted would be greater following SC exposure. There were no significant differences in responses to any of the items for either the SCs (Table 2) or the non-SCs (not shown). Of note, overall craving reports to SCs approached significance and were positive in 9-repeats and negative in 10/10-repeats (Table 2, item 3, $p<0.11$ ).

\section{Imaging Results}

For comparisons between conditions and/or groups and regression analyses only clusters with voxels having a height threshold exceeding $p<0.001$ (uncorrected), an extent threshold of 20 contiguous voxels, and significant at the cluster level are reported. Coordinates are in MNI as provided by SPM, however, can be converted to Tailarach coordinates (http://www.mrc-cbu.cam.ac.uk/Imaging/mnispace.html). Coordinates listed are those chosen from the suprathreshold voxel of each ROI using the Duvernoy Brain Atlas as a reference (Duvernoy, 1999).

Smoking cue reactivity in 9-repeats vs 10/10-repeats. In comparison to 10/10-repeats, 9-repeats exhibited significantly greater activity in response to SCs vs non-SCs bilaterally in the OFC and parahippocampus; in the left VS/ ventral pallidal (VP) region, and dorsolateral PFC; and in the right ventral medial PFC, fusiform gyrus, and superior temporal and frontal gyri. Activity was reduced in the right superior frontal gyrus. Representative axial and coronal sections coregistered to the MNI brain are shown in Figure 1. See Table 3 for $T$-values and MNI coordinates, as provided by SPM.

Smoking cue reactivity in separate allelic groups. The brain's response to SCs was also examined separately in 9repeats and 10/10-repeats. 9-repeats exhibited enhanced activity bilaterally in OFC, VS/VP, and parahippocampal gyrus; and in left amygdala, anterior cingulate, and superior frontal gyrus. Notably, activity was decreased in 10/10repeats bilaterally in the OFC and in the left VS/VP, ventral

Table 2 Table of Craving and Withdrawal Questionnaire Scores Generated from the Changes Elicited by the Smoking Stimuli (PostScores-Pre-Scores) in 9-Repeats $(N=10)$ vs 10/10-Repeats $(N=9)$

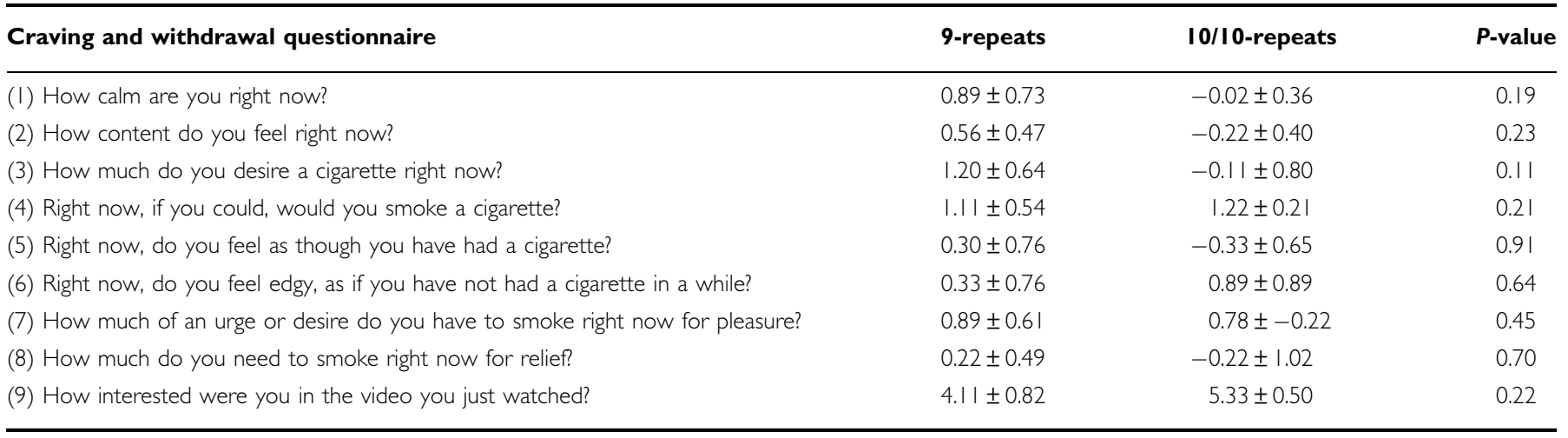

Each question was preceded by the phrase 'On a scale from I to $7 \ldots$..' Data were analyzed using two-tailed $t$-tests for all items except item 3. There were no significant differences across groups in any of the nine items. 
Table 3 Brain Activity in 9-Repeat vs 10/10-Repeats, 9-Repeats, and 10/10-Repeats

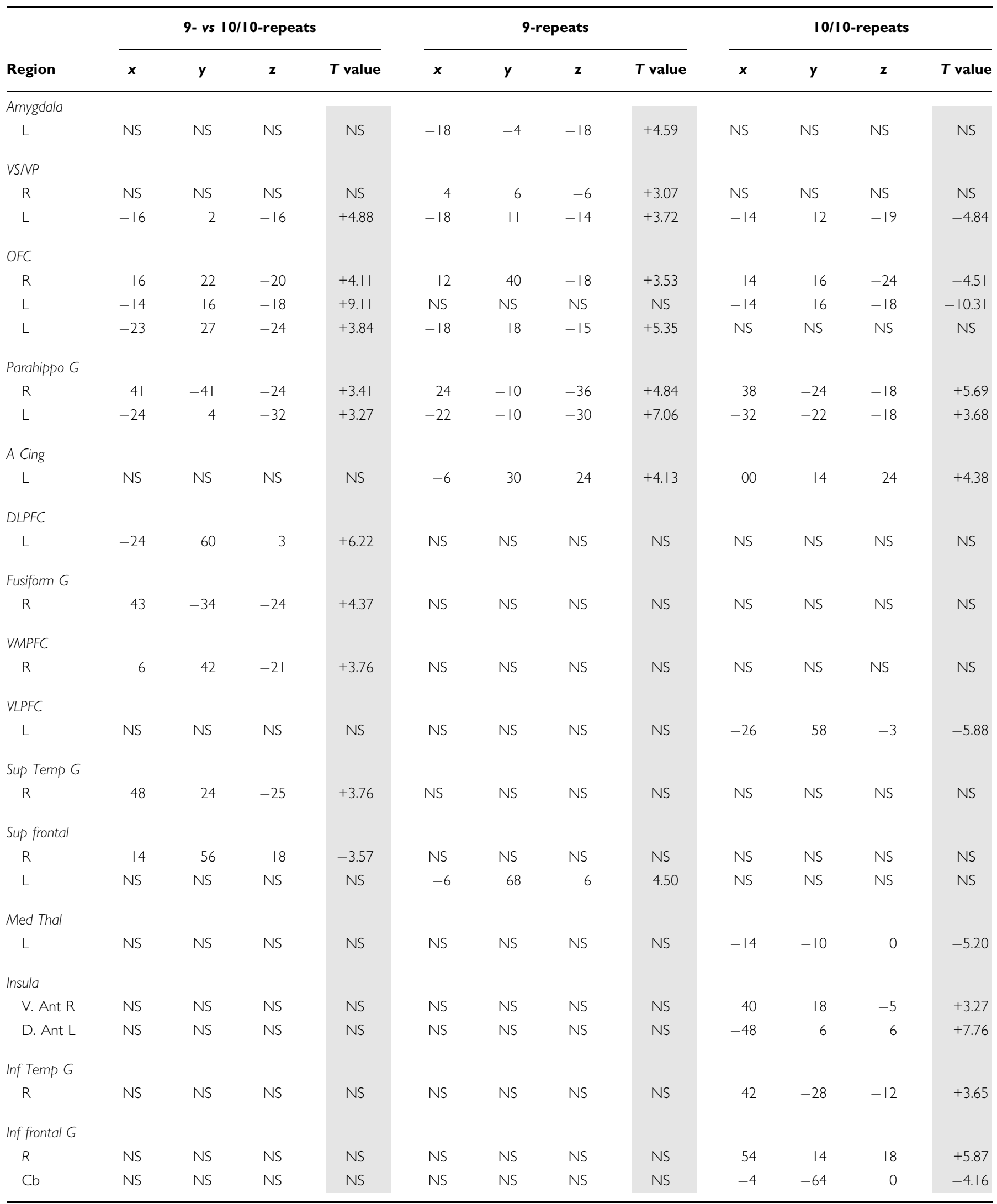

Abbreviations: A Cing, anterior cingulate; Ant, anterior; Cb, cerebellum; D, dorsal; DLPFC, dorsolateral prefrontal cortex; G, gyrus; Inf, inferior; L, left; Med Thal, medial thalamus; Parahippo, parahippocampal; N, nonsignificant; R, right; Sup, superior; Temp, temporal; V, ventral; VLPFC, ventral lateral prefrontal cortex; VMPFC, ventral medial prefrontal cortex; VP, ventral pallidal; VS, ventral striatum.

Coordinates are in MNI. 
lateral prefrontal cortex, medial thalamus and cerebellum; and was greater bilaterally in the insula and parahippocampal gyrus and in the right inferior temporal and left inferior frontal gyri and anterior cingulate (Table 3; Figure 1). There were no differences between groups in resting baseline blood flow at this threshold indicating that the differences were unrelated to variation in neurophysiology present before cue exposure. There were no differences between groups in brain responses to non-SCs.

Brain and behavioral correlates by genotype. Linear regression analyses were conducted separately for each genotype on the change in craving scores to SCs and brain activity at each voxel (Figure 2). Although brain activity and craving was reduced following SC exposure in 10/10-repeats, strong correlations between subjective craving responses and brain activity were observed in the left OFC, $r^{2}=0.86, T=5.92$; left VS, $r^{2}=0.85, T=3.81$; right $\mathrm{VS}, T=3.72, r^{2}=0.86$; anterior cingulate (rostral supragenual region), $r^{2}=0.89, T=4.72$; left parahippocampal gyrus, $r^{2}=0.85, T=4.46$; left insula, $r^{2}=0.85, T=5.15 ;$ and right insula $r^{2}=0.85, T=3.40$ (anterior ventral). In contrast, there were no significant correlations between brain responses and craving in 9repeats; however, a trend was observed in VS $\left(r^{2}=0.29\right)$. This effect was statistically significant between groups at each region ( $T$-values ranged from 4.42 to 5.69 at $p<0.01$, 20 contiguous voxels). Notably, the brain responses in individual 9-repeat subjects were inconsistent and quite variable across regions. For example, the smoker with a change in craving score of ' 2 ' and who had the greatest brain activity compared to other smokers in the VS is not the same individual whose score was also '2' and had the greatest perfusion in the OFC. Also, one subject had extremely high activity in the OFC. The lack of a relationship between craving and brain activity was still present using Spearman's $\rho$ correlation analysis and when that subject was excluded from the analysis. Brain/behavioral correlations were not observed in any other regions in either group.

\section{DISCUSSION}

\section{Summary of Results}

Here we report that genetic variation in dopaminergic transmission contributes to the brain and behavioral responses elicited by SCs. We observed increased brain activity during exposure to SC $v s$ non-SCs in smokers carrying at least one 9-repeat-VNTR allele of the DAT SCL6A3 gene (9-repeats) in comparison to 10-repeat-VNTR homozygotes (10/10-repeats) in the interconnected OFC/ VS/VP. Subsequent analyses after grouping smokers by genotype produced quite different patterns of activity. Brain activity was heightened bilaterally in the OFC/VS/VP in 9repeats and reduced in the same regions in 10/10-repeats. Further, no significant correlations were found between brain activity and craving in 9-repeats, whereas neural activity strongly correlated with craving in several a priori regions in 10/10-repeats: OFC, VS, anterior cingulate, parahippocampal gyrus, and insula.

As expected, 9-repeats exhibited increased neural activity to SCs in reward-related circuitry (amygdala, VS/VP, parahippocampal gyrus, OFC, and anterior cingulate) compared to 10/10-repeats. These results are analogous to our earlier findings characterizing the neural correlates of withdrawal-free SC reactivity (Franklin et al, 2007). To delineate further the influence of DAT genotype on cue reactivity, separate analyses were conducted on 9-repeats and 10/10-repeats. We expected SCs to elicit increased activity in reward-related circuitry in both groups, albeit greater in 9-repeats. Contrary to expectation, we observed dramatically different brain activation patterns in the separate groups. 9-repeats exhibited increases in activity in amygdala, OFC/VS/VP, parahippocampal gyrus, and anterior cingulate whereas $10 / 10$-repeats revealed equivalent but opposite responses in VS/VP/OFC and increased activity in parahippocampus, anterior cingulate, and insula.

\section{Possible Role for the DAT in Cue Reactivity}

The repetitive intermittent surges in VS DA stimulated by nicotine and other addictive drugs strengthen stimulusdrug associations and the pathways that mediate them (Balfour et al, 2000; Di Chiara et al, 1999; Pontieri et al, 1998). Thus, discrete stimuli or contexts predictive of drug availability acquire powerful incentive properties and motivate drug-seeking behavior (Parkinson et al, 2002; Stuber et al, 2005). The phasically released DA in response to rewards and their predictors is rapidly removed by the DAT. The 9-repeat-VNTR allele has been associated with lower DAT expression in the VS in molecular studies (Fuke et al, 2001; Mill et al, 2002; VanNess et al, 2005); however, in vivo studies are less consistent. Studies quantifying DAT protein availability report that 9-repeats exhibit higher striatal availability in healthy subjects (van Dyck et al, 2005); lower availability in alcoholics and controls, irrespective of alcohol dependence (Heinz et al, 2000); and no change in DAT density in schizophrenics and controls, also irrespective of schizophrenia (Martinez et al, 2001). In our view, DAT availability does not imply DAT function and we suggest the increased perfusion in 9-repeats during SC exposure in the VS and related circuitry is indirect evidence of reduced DAT activity and support our working hypothesis that extending the time between DA release and DA reuptake increases the incentive salience of smoking stimuli

Figure I Representative $\mathrm{MMR}$ axial and coronal brain slices analyzed in SPM2 and displayed in MRIcro (two-tailed) overlaid on the Montreal Neurologica Institute (MNI) brain showing brain activity to the smoking cues greater than to the nonsmoking cues in 9-repeats vs I0/I0-repeats, 9-repeats, and I0/I0repeats. T-values range from negative $(-)$ I0.3I to positive $(+) 9.1$ I. Crosshairs are centered on the suprathreshold voxel of representative significant clusters. Note that not all regions showing significance are centered under crosshairs. See Table 3 for a complete list of coordinates and T-values of regions showing significant activations in response to smoking cues. Images are displayed neurologically (left is left). 

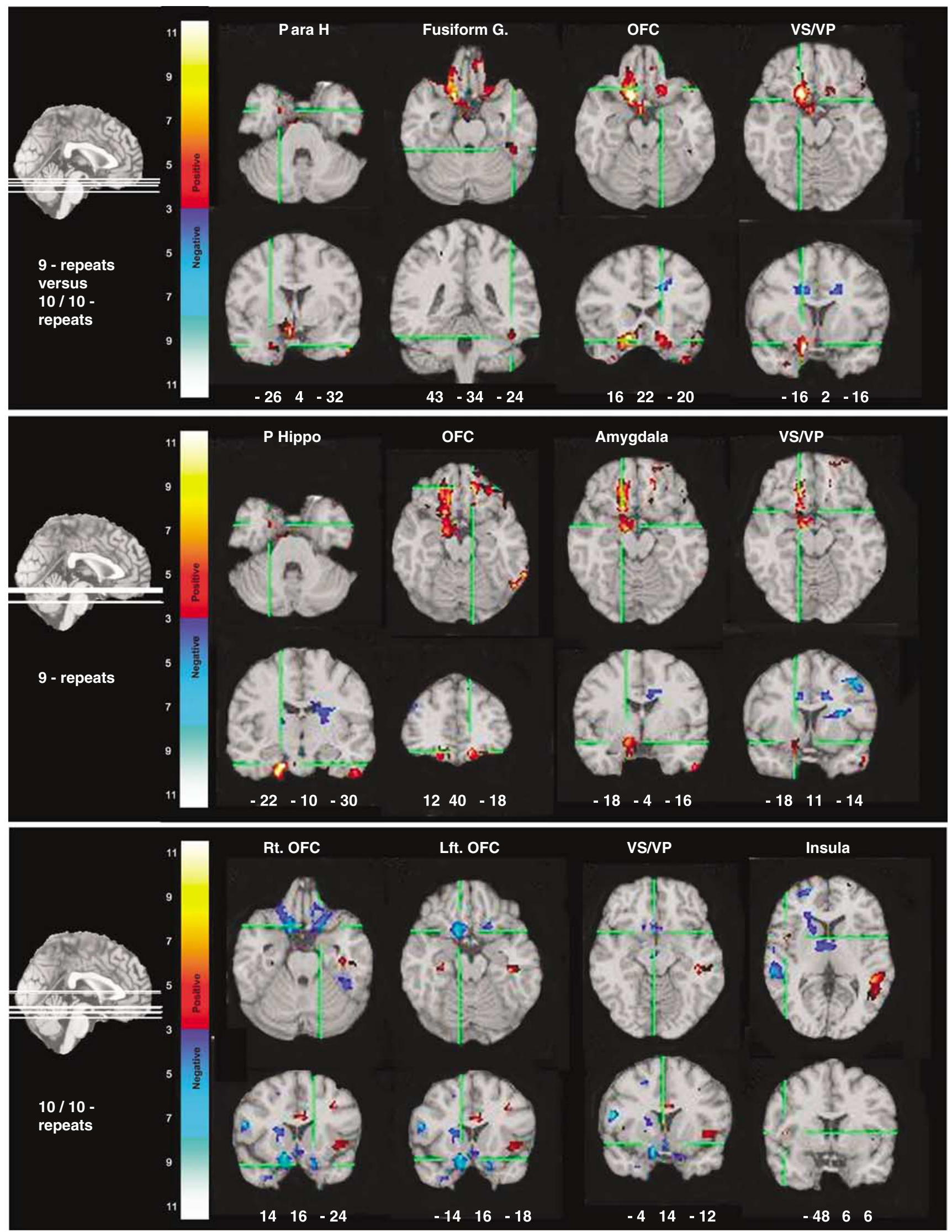
Orbitofrontal cortex

( - 48 34-12)

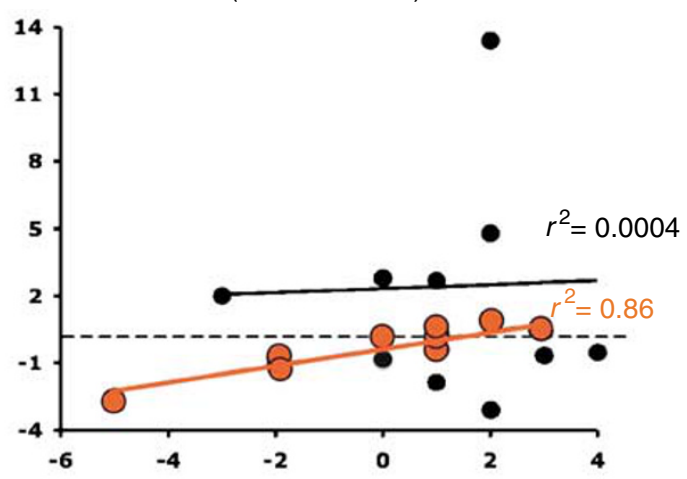

Ventral striatum

$\left(\begin{array}{ll}-20 & 12-6\end{array}\right)$

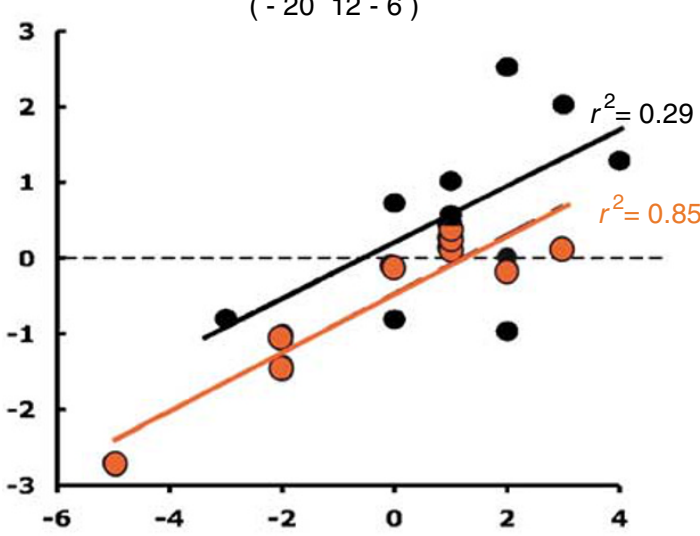

Change in craving

9-repeats

10-repeats
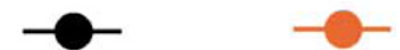

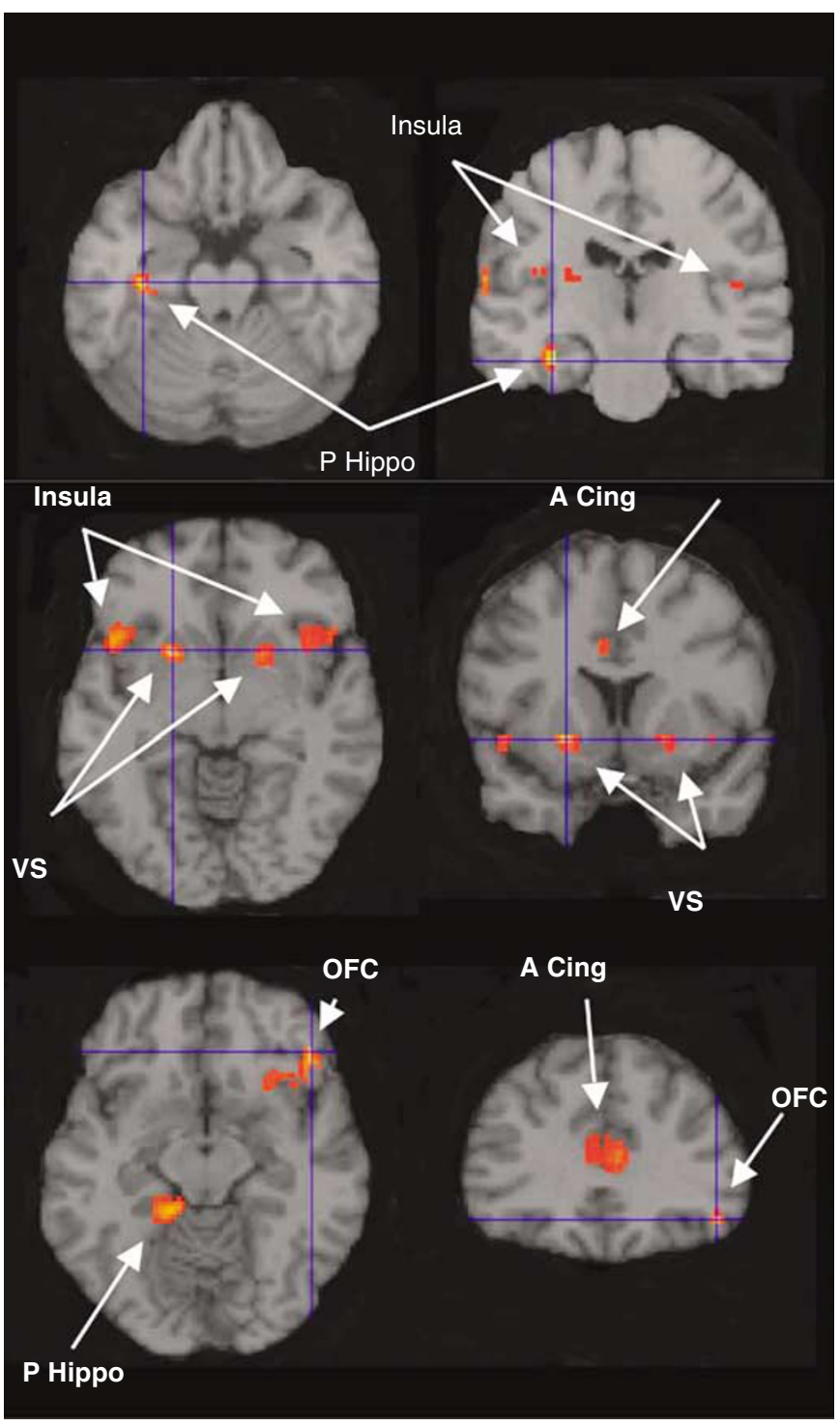

Figure 2 Graphs of individual perfusion $\mathrm{fMRI}$ responses ( $\beta$-coefficients) as a function of the change in craving elicited by the smoking cues in the orbitofrontal cortex (OFC) and ventral striatum (VS). Strong correlations in 10/I0-repeats contrast with weak or insignificant correlations in 9-repeats. Imaging data were analyzed in SPM2 and is displayed in MRIcro overlaid on the Montreal Neurological Institute (MNI) brain. As shown in representative brain slices, correlations with craving in 10/10-repeats were also observed in anterior cingulate, $r^{2}=0.89$; right ventral striatum, $r^{2}=0.85$; left parahippocampal gyrus, $r^{2}=0.85$; and bilaterally in the insula, $r^{2}=0.8 \mathrm{I}$ on right and 0.85 on left. Coordinates are in MNI. Note that the abscissas are adjusted in relation to the intensity of perfusion in each region. Slices are displayed neurologically in (left is left). T-values are shown in the graph (right) and ranged from 4.42 to 5.69 at $p<0.01$, 20 contiguous voxels.

and may increase the probability of relapse associated with cue exposure.

\section{Interpretation of Results}

Differences between groups in underlying motivational processes, possibly genetically mediated, may partially explain the differences in brain activation. As the two primary motivators to smoke are pharmacological withdrawal from nicotine and exposure to smoking-related stimuli, and as smokers were satiated prior to cue sessions, we speculate that the pharmacological and physiological state produced by recent smoking eliminated the power of the cues to elicit a brain or behavioral response in 10/10- repeats, whose smoking behavior may be motivated to a larger extent by the absence of nicotine in the brain. As the OFC is involved in assigning the relative motivational significance to rewards and their signals (Salzman et al, 2007), a reduction in activity in that region may signal reduced cue value in the sated condition. Alternatively, activity in the OFC and related regions subserving motivational responses to emotional stimuli may be sustained even in the sated state in 9-repeats with a less active or less available form of the DAT.

The above interpretation may explain some of the variance but does not address the finding of a decreased response in VS/VP/OFC in 10/10-repeats. It is unlikely that they were uninterested or able to ignore the SCs as the 
parahippocampal region, important in episodic memory (conscious recall of autobiographical events), was strongly activated in both groups, and subjective responses assessing cue interest did not differ between groups.

Were SCs aversive to 10/10-repeats in the satiated condition? It has been shown that even chocolate, as palatable as it may be to professed chocolate lovers, becomes increasingly unpleasant as more of it is consumed, which correlates dose dependently with changes in the location and direction of neural activity (Small et al, 2001). Further, 10/10-repeats exhibited strong bilateral activation in the insula, not observed in 9-repeats. This is consistent with a wealth of literature wherein insula activation is associated with increased feelings of disgust to aversive stimuli (Schafer et al, 2005; Schienle et al, 2002; Stark et al, 2007; Wright et al, 2004). This interpretation is supported by recent work in our laboratory with cocaine-addicted patients wherein we demonstrated a positive correlation between brain activation bilaterally in the insula to $33 \mathrm{~ms}$ unseen aversive targets and negative affective bias to visible versions of the same stimuli in an off-magnet task (Childress et al, 2008). It is also supported by the finding here of an overall decrease in craving elicited by SCs only in 10/10repeats. There is also an extensive literature wherein heightened insula activity is implicated in mediating responses to appetitive stimuli, including our previous study examining responses to smoking stimuli explicitly designed to be highly appetitive to smokers (Bjork et al, 2004; Franklin et al, 2007; Hinton et al, 2004; Wang et al, 2004). As the insula is a large and heterogeneous structure and is implicated in mediating both aversive and appetitive responses, its continued study focusing on discrete regional activity is warranted before a clear interpretation can be made. The addition of an affective bias task will test the above hypothesis in future work.

Although no significant correlations with craving and brain response was observed in 9-repeats, 10/10-repeats exhibited robust correlations between brain and behavior in several reward-related regions. We tentatively suggest that processing in the frontostriatal regulatory network is disturbed in 9-repeats such that they are less able to correctly label their craving experience. The inability to label craving may confer enhanced vulnerability to the effects of cues and therefore increased probability of relapse in their presence. In other words not being able to identify feelings correctly may make it harder to control behavior. Although not tested here and purely speculative at this juncture, the inability to describe or identify the craving state may reflect an underlying and genetically mediated personality trait that generalizes to other emotions such that 9-repeats are exhibiting an 'alexithymic' response to cues. Alexythymia is a personality trait characterized by deficits in cognitive processing of emotions and is highly prevalent in various psychological disorders (Alvarez and Shipko, 1991; Shipko et al, 1983) including drug addiction (Taylor et al, 1990). Alexithymic individuals are deficient in understanding and describing emotions and are at high risk for poor treatment response using conventional methods (Haviland et al, 2000). In support of this theory, the neural responses in 9-repeats concur with those of a PET study comparing alexithymic and normal responses to emotional stimuli. As in 9-repeats, alexithymics had less activation in superior frontal gyrus and anterior cingulate and greater activation in ventral medial prefrontal gyrus (Karlsson et al, 2008). However, the literature examining brain correlates of alexithymia is sparse and somewhat inconsistent, with the exception of a general consensus on the lack of activation in the anterior and superior frontal gyri in alexithymic individuals (Berthoz et al, 2002; Huber et al, 2002; Kano et al, 2003).

An alternative explanation for the lack of association between brain activity and craving in 9-repeats is that the cues had induced a maximal response in brain blood flow to reward-related regions. This interpretation is unlikely as examination of the pattern of activation in individual 9repeats is quite variable across subjects and across regions. This interpretation is further undermined as pre-cue craving scores were similar in both groups. Nevertheless, this possibility cannot be ruled out.

Notably, overall craving difference scores to the SCs approached significance and were positive in 9-repeats and negative in 10/10-repeats. These same sets of cues elicited craving in a previous study, demonstrating that the SCs do elicit a robust subjective craving response in sated smokers (Franklin et al, 2007). Although the brain's response to emotionally laden processes is not under subjective control, neurophysiological measures are presumed to be more direct and more sensitive than subjective measures of the same processes, requiring much smaller sample sizes to demonstrate effects (Hariri et al, 2006). Our previous study had twice the number of subjects, which appears to be necessary to achieve significant overall craving difference scores in sated smokers. There were also no differences in other behavioral measures such as cigarette dependence as measured by the FTND or number of cigarettes smoked per day. Again, this may be a function of small sample size. Alternatively, genotype may not predict the degree of dependence, cigarettes smoked per day or even smoking cessation outcome. It may be related to differential underlying motivations to continued smoking and relapse as discussed above, and if true, could aid in our efforts to reduce relapse rates by using genotype to predict medication response. For example, if 9-repeats' smoking behavior is affected more by SCs and less by WD symptoms, nicotine replacement therapy may not be a viable treatment option.

\section{Study Limitations}

These results should be interpreted with caution given the modest sample size. In addition, the use of probands of various ethnic backgrounds might lead to issues of population stratification as global population studies have demonstrated varying frequencies of the two alleles (Gelernter et al, 1998; Palmatier et al, 1999). Future studies will have to be carefully matched for ethnic background and might have to include genomic controls to address issues of population stratification.

Further investigation into the genetics underlying DA signaling in reactivity to SCs is warranted since it is likely that additional polymorphisms contribute to different responses. Indeed, a DA D4 receptor subtype polymorphism has been implicated in influencing responses to SCs (Hutchison et al, 2002; McClernon et al, 2007). The DA 
receptor subtype 2 (DRD2) is also a likely candidate as genetic load has been associated with an increased risk of substance abuse and other addictive behaviors, including smoking (Batra et al, 2003; Comings et al, 1996; Noble et al, 1994), however see (Bierut et al, 2000; Singleton et al, 1998). In addition, genetic variance in the DRD2 Taql RFLP and the DAT SLC6A3 had an additive effect on subjective craving scores generated by SC exposure (Erblich et al, 2005). Interactions between dopaminergic polymorphisms may also be important. For instance, individual differences in VS sensitivity to reward were partly explained by an epistatic gene-gene interaction between the SLC6A3 and the catechol-omethyltransferase (COMT) genes (Yacubian et al, 2007). Because of these and other studies we will broaden our examination to determine whether SC reactivity is modulated by genetic variance in the DRD2 and DRD4 receptor subtypes and in COMT.

\section{Conclusions}

Undeniably, dependence on cigarettes is the number one cause of premature death and exacts a massive economic burden on families and society as a whole. Though relapse to smoking and other psychoactive drugs is multidetermined, a commonly cited state that precedes relapse is cue-induced craving. It is imperative that we continue to strive to understand the brain mechanisms underlying craving and its link to relapse. These results support our hypothesis that the brain's response to SCs is partially genetically mediated through variance originating in dopaminergic components.

They point to an underlying preexisting mechanism in the etiology of addiction processes by providing evidence that the neural and behavioral responses to smoking-related stimuli are mediated by at least one dopaminergic gene variant. These results are a significant contribution to the field of cigarette and other addictions, as they may prove useful in identifying endophenotypes, which may have immediate implications for clinical outcome and for medications development. Further, they indicate that the combined use of neuroimaging and genomics offers the potential to develop predictive markers of relapse vulnerability and identify novel targets for individualized treatment.

\section{ACKNOWLEDGEMENTS}

This work was supported by NIH grants DA015149, K01 DA 015426-011A1, 5-P60-DA-005186-18, and NS045839, BCS0224007, RR02305, 1K08MH080372-01A1 VA VISN 4 MIRECC, and the GCRC of the University of Pennsylvania. We acknowledge the nursing staff at the University of Pennsylvania Addiction Treatment Research for conducting physical evaluations. We also thank our clinicians Anita Hole PhD, Jesse Suh, PsyD, and Marta MacDougal, PsyD for conducting the psychological evaluations. And third, we take this opportunity to thank the MRI technicians at the Hospital of the University of Pennsylvania for conducting the scanning sessions.

\section{DISCLOSURE/CONFLICT OF INTEREST}

Dr JA Detre has received royalties for the commercial licensure of ASL perfusion fMRI. None of the other authors have reported any potential conflicts of interest.

\section{REFERENCES}

Aguirre GK, Detre JA, Wang J (2005). Perfusion fMRI for functional neuroimaging. Int Rev Neurobiol 66: 213-236.

Alvarez WA, Shipko S (1991). Alexithymia and posttraumatic stress disorder. J Clin Psychiatry 52: 317-319.

Baillie AJ, Mattick RP, Hall W (1995). Quitting smoking: estimation by meta-analysis of the rate of unaided smoking cessation. Aust J Public Health 19: 129-131.

Baker TB, Morse E, Sherman JE (1986). The motivation to use drugs: a psychobiological analysis of urges. Nebr Symp Motiv 34: 257-323.

Balfour DJ, Wright AE, Benwell ME, Birrell CE (2000). The putative role of extra-synaptic mesolimbic dopamine in the neurobiology of nicotine dependence. Behav Brain Res 113: 73-83.

Batra V, Patkar AA, Berrettini WH, Weinstein SP, Leone FT (2003). The genetic determinants of smoking. Chest 123: 1730-1739.

Berthoz S, Artiges E, Van De Moortele PF, Poline JB, Rouquette S, Consoli SM et al (2002). Effect of impaired recognition and expression of emotions on frontocingulate cortices: an fMRI study of men with alexithymia. Am J Psychiatry 159: 961-967.

Bierut LJ, Rice JP, Edenberg HJ, Goate A, Foroud T, Cloninger CR et al (2000). Family-based study of the association of the dopamine D2 receptor gene (DRD2) with habitual smoking. Am J Med Genet 90: 299-302.

Bjork JM, Knutson B, Fong GW, Caggiano DM, Bennett SM, Hommer DW (2004). Incentive-elicited brain activation in adolescents: similarities and differences from young adults. J Neurosci 24: 1793-1802.

Boileau I, Dagher A, Leyton M, Welfeld K, Booij L, Diksic M et al (2007). Conditioned dopamine release in humans: a positron emission tomography [11C]raclopride study with amphetamine. J Neurosci 27: 3998-4003.

Brody AL, Mandelkern MA, Olmstead RE, Scheibal D, Hahn E, Shiraga S et al (2006). Gene variants of brain dopamine pathways and smoking-induced dopamine release in the ventral caudate/nucleus accumbens. Arch Gen Psychiatry 63: 808-816.

Cancer Facts and Figures (2007). Presented by the American Cancer Society: Atlanta, Georgia. http://www.cancer.org/docroot/ PRO/content/PRO_1_1_Cancer_Statistics_2007_Presentation.asp

Centers for Disease Control and Prevention (1993). Cigarette smoking-attributable mortality and years of potential life lost-United States, 1990. Morbidity and Mortality Weekly Report [serial online]. 42: 645-648. Available from: http:// www.cdc.gov/mmwr/preview/mmwrhtml/00021441.htm

Childress AR, Ehrman RN, Wang Z, Li Y, Sciortino N, Hakun J et al (2008). Prelude to passion: limbic activation by 'unseen' drug and sexual cues. PLOS ONE 3: e1506.

Childress AR, McElgin W, Mozley PD, Fitzgerald J, Reivich M, O'Brien CP (1999). Limbic activation during cue-induced cocaine craving. Am J Psychiatry 156: 11-18.

Comings DE, Ferry L, Bradshaw-Robinson S, Burchette R, Chiu C, Muhleman D (1996). The dopamine D2 receptor (DRD2) gene: a genetic risk factor in smoking. Pharmacogenetics 6: 73-79.

Corrigall WA, Franklin KB, Coen KM, Clarke PB (1992). The mesolimbic dopaminergic system is implicated in the reinforcing effects of nicotine. Psychopharmacology (Berl) 107: 285-289.

Detre JA, Leigh JS, Williams DS, Koretsky AP (1992). Perfusion imaging. Magn Reson Med 23: 37-45.

Detre JA, Wang J (2002). Technical aspects and utility of fMRI using BOLD and ASL. Clin Neurophysiol 113: 621-634. 
Di Chiara G (2000). Role of dopamine in the behavioural actions of nicotine related to addiction. Eur J Pharmacol 393: 295-314.

Di Chiara G, Imperato A (1988). Drugs abused by humans preferentially increase synaptic dopamine concentrations in the mesolimbic system of freely moving rats. Proc Natl Acad Sci USA 85: 5274-5278.

Di Chiara G, Tanda G, Bassareo V, Pontieri F, Acquas E, Fenu S et al (1999). Drug addiction as a disorder of associative learning. Role of nucleus accumbens shell/extended amygdala dopamine. Ann N Y Acad Sci 877: 461-485.

Di Ciano P, Blaha CD, Phillips AG (1998). The relation between dopamine oxidation currents in the nucleus accumbens and conditioned increases in motor activity in rats following repeated administration of d-amphetamine or cocaine. Eur $J$ Neurosci 10: 1113-1120.

Droungas A, Ehrman RN, Childress AR, O'Brien CP (1995). Effect of smoking cues and cigarette availability on craving and smoking behavior. Addict Behav 20: 657-673.

Duvauchelle CL, Ikegami A, Castaneda E (2000). Conditioned increases in behavioral activity and accumbens dopamine levels produced by intravenous cocaine. Behav Neurosci 114: 1156-1166.

Duvernoy HM (1999). The Human Brain: Surface, Three-Dimensional Sectional Anatomy with Magnetic Resonance Imaging and Blood Supply, 2nd edn. Springer-Verlag Wien: New York City.

Erblich J, Lerman C, Self DW, Diaz GA, Bovbjerg DH (2005). Effects of dopamine D2 receptor (DRD2) and transporter (SLC6A3) polymorphisms on smoking cue-induced cigarette craving among African-American smokers. Mol Psychiatry 10: 407-414.

Fagerstrom KO, Schneider NG (1989). Measuring nicotine dependence: a review of the Fagerstrom Tolerance Questionnaire. J Behav Med 12: 159-182.

Floyd TF, Ratcliffe SJ, Wang J, Resch B, Detre JA (2003). Precision of the CASL-perfusion MRI technique for the measurement of cerebral blood flow in whole brain and vascular territories. J Magn Reson Imaging 18: 649-655.

Franklin TR, Druhan JP (2000a). Expression of Fos-related antigens in the nucleus accumbens and associated regions following exposure to a cocaine-paired environment. Eur $J$ Neurosci 12: 2097-2106.

Franklin TR, Druhan JP (2000b). Involvement of the nucleus accumbens and medial prefrontal cortex in the expression of conditioned hyperactivity to a cocaine-associated environment in rats. Neuropsychopharmacology 23: 633-644.

Franklin TR, Wang Z, Wang J, Sciortino N, Harper D, Li Y et al (2007). Limbic activation to cigarette smoking cues independent of nicotine withdrawal: a perfusion fMRI study. Neuropsychopharmacology 32: 2301-2309.

Fuke S, Suo S, Takahashi N, Koike H, Sasagawa N, Ishiura S (2001). The VNTR polymorphism of the human dopamine transporter (DAT1) gene affects gene expression. Pharmacogenomics $J$ 1: 152-156.

Gelernter J, Kranzler H, Lacobelle J (1998). Population studies of polymorphisms at loci of neuropsychiatric interest (tryptophan hydroxylase (TPH), dopamine transporter protein (SLC6A3), D3 dopamine receptor (DRD3), apolipoprotein E (APOE), mu opioid receptor (OPRM1), and ciliary neurotrophic factor (CNTF)). Genomics 52: 289-297.

Gratton A, Wise RA (1994). Drug- and behavior-associated changes in dopamine-related electrochemical signals during intravenous cocaine self-administration in rats. J Neurosci 14: 4130-4146.

Hariri AR, Drabant EM, Weinberger DR (2006). Imaging genetics: perspectives from studies of genetically driven variation in serotonin function and corticolimbic affective processing. Biol Psychiatry 59: 888-897.

Haviland MG, Warren WL, Riggs ML (2000). An observer scale to measure alexithymia. Psychosomatics 41: 385-392.
Heath AC, Madden PAF (1995). Genetic influences on smoking behavior. In: Behavior Genetic Approaches in Behavioral Medicine, Turner JR, Cardon LR, Hewitt JK (eds). Plenum Press: New York. 45-65.

Heinz A, Goldman D, Jones DW, Palmour R, Hommer D, Gorey JG et al (2000). Genotype influences in vivo dopamine transporter availability in human striatum. Neuropsychopharmacology 22: 133-139.

Hinton EC, Parkinson JA, Holland AJ, Arana FS, Roberts AC, Owen AM (2004). Neural contributions to the motivational control of appetite in humans. Eur J Neurosci 20: 1411-1418.

Huber M, Herholz K, Habedank B, Thiel A, Müller-Küppers M, Ebel $\mathrm{H}$ et al (2002). [Different patterns of regional brain activation during emotional stimulation in alexithymics in comparison with normal controls]. Psychother Psychosom Med Psychol 52: 469-478.

Hughes JR (2007). Effects of abstinence from tobacco: valid symptoms and time course. Nicotine Tob Res 9: 315-327.

Hughes JR, Shiffman S, Callas P, Zhang J (2003). A meta-analysis of the efficacy of over-the-counter nicotine replacement. Tob Control 12: 21-27.

Hutchison KE, LaChance H, Niaura R, Bryan A, Smolen A (2002). The DRD4 VNTR polymorphism influences reactivity to smoking cues. J Abnorm Psychol 111: 134-143.

Jaber M, Jones S, Giros B, Caron MG (1997). The dopamine transporter: a crucial component regulating dopamine transmission. Mov Disord 12: 629-633.

Kano M, Fukudo S, Gyoba J, Kamachi M, Tagawa M, Mochizuki H et al (2003). Specific brain processing of facial expressions in people with alexithymia: an H2 15O-PET study. Brain 126: $1474-1484$

Karlsson H, Naatanen P, Stenman H (2008). Cortical activation in alexithymia as a response to emotional stimuli. $\mathrm{Br} J$ Psychiatry 192: $32-38$.

Lahiri DK, Nurnberger Jr JI (1991). A rapid non-enzymatic method for the preparation of HMW DNA from blood for RFLP studies. Nucleic Acids Res 19: 5444

Martinez D, Gelernter J, Abi-Dargham A, van Dyck CH, Kegeles L, Innis $\mathrm{RB}$ et al (2001). The variable number of tandem repeats polymorphism of the dopamine transporter gene is not associated with significant change in dopamine transporter phenotype in humans. Neuropsychopharmacology 24: 553-560.

Mash DC, Pablo J, Ouyang Q, Hearn WL, Izenwasser S (2002). Dopamine transport function is elevated in cocaine users. J Neurochem 81: 292-300.

McBride D, Barrett SP, Kelly JT, Aw A, Dagher A (2006). Effects of expectancy and abstinence on the neural response to smoking cues in cigarette smokers: an fMRI study. Neuropsychopharmacology 31: 2728-2738.

McClernon FJ, Hutchison KE, Rose JE, Kozink RV (2007). DRD4 VNTR polymorphism is associated with transient fMRI-BOLD responses to smoking cues. Psychopharmacology (Berl) 194: 433-441.

Mill J, Asherson P, Browes C, D'Souza U, Craig I (2002). Expression of the dopamine transporter gene is regulated by the $3^{\prime}$ UTR VNTR: evidence from brain and lymphocytes using quantitative RT-PCR. Am J Med Genet 114: 975-979.

Noble EP, St Jeor ST, Ritchie T, Syndulko K, St Jeor SC, Fitch RJ et al (1994). D2 dopamine receptor gene and cigarette smoking: a reward gene? Med Hypotheses 42: 257-260.

Palmatier MA, Kang AM, Kidd KK (1999). Global variation in the frequencies of functionally different catechol- $O$-methyltransferase alleles. Biol Psychiatry 46: 557-567.

Parkinson JA, Dalley JW, Cardinal RN, Bamford A, Fehnert B, Lachenal G et al (2002). Nucleus accumbens dopamine depletion impairs both acquisition and performance of appetitive Pavlovian approach behaviour: implications for mesoaccumbens dopamine function. Behav Brain Res 137: 149-163. 
Penny W, Friston K (2003). Mixtures of general linear models for functional neuroimaging. IEEE Trans Med Imaging 22: 504-514.

Pontieri FE, Passarelli F, Calo L, Caronti B (1998). Functional correlates of nicotine administration: similarity with drugs of abuse. J Mol Med 76: 193-201.

Salzman CD, Paton JJ, Belova MA, Morrison SE (2007). Flexible neural representations of value in the primate brain. Ann NY Acad Sci 1121: 336-354.

Schafer A, Schienle A, Vaitl D (2005). Stimulus type and design influence hemodynamic responses towards visual disgust and fear elicitors. Int J Psychophysiol 57: 53-59.

Schienle A, Stark R, Walter B, Blecker C, Ott U, Kirsch P et al (2002). The insula is not specifically involved in disgust processing: an fMRI study. Neuroreport 13: 2023-2026.

Sheehan B, Lecrubier Y, Sheehan K (1998). The mini international neuropsychiatric interview (MINI): the development and validation of structured diagnostic interview for DSM-IV and ICD-10. J Clin Psychiatry 59: 22-33.

Shiffman S, Paty JA, Gnys M, Kassel JA, Hickcox M (1996). First lapses to smoking: within-subjects analysis of real-time reports. J Consult Clin Psychol 64: 366-379.

Shipko S, Alvarez WA, Noviello N (1983). Towards a teleological model of alexithymia: alexithymia and post-traumatic stress disorder. Psychother Psychosom 39: 122-126.

Singleton AB, Thomson JH, Morris CM, Court JA, Lloyd S, Cholerton $S$ (1998). Lack of association between the dopamine $\mathrm{D} 2$ receptor gene allele $\mathrm{DRD} 2{ }^{\star} \mathrm{A} 1$ and cigarette smoking in a United Kingdom population. Pharmacogenetics 8: 125-128.

Small DM, Zatorre RJ, Dagher A, Evans AC, Jones-Gotman M (2001). Changes in brain activity related to eating chocolate: from pleasure to aversion. Brain 124: 1720-1733.

Stark R, Zimmermann M, Kagerer S, Schienle A, Walter B, Weygandt $M$ et al (2007). Hemodynamic brain correlates of disgust and fear ratings. Neuroimage 37: 663-673.

Stuber GD, Roitman MF, Phillips PE, Carelli RM, Wightman RM (2005). Rapid dopamine signaling in the nucleus accumbens during contingent and noncontingent cocaine administration. Neuropsychopharmacology 30: 853-863.

Taylor GJ, Parker JD, Bagby RM (1990). A preliminary investigation of alexithymia in men with psychoactive substance dependence. Am J Psychiatry 147: 1228-1230. van Dyck CH, Malison RT, Jacobsen LK, Seibyl JP, Staley JK, Laruelle $\mathrm{M}$ et al (2005). Increased dopamine transporter availability associated with the 9-repeat allele of the SLC6A3 gene. J Nucl Med 46: 745-751.

Vandenbergh DJ, Bennett CJ, Grant MD, Strasser AA, O'Connor R, Stauffer RL et al (2002). Smoking status and the human dopamine transporter variable number of tandem repeats (VNTR) polymorphism: failure to replicate and finding that never-smokers may be different. Nicotine Tob Res 4: 333-340.

Vandenbergh DJ, Persico AM, Hawkins AL, Griffin CA, Li X, Jabs EW et al (1992). Human dopamine transporter gene (DAT1) maps to chromosome 5p15.3 and displays a VNTR. Genomics 14: 1104-1106.

VanNess SH, Owens MJ, Kilts CD (2005). The variable number of tandem repeats element in DAT1 regulates in vitro dopamine transporter density. BMC Genet 6: 55 .

Volkow ND, Wang GJ, Telang F, Fowler JS, Logan J, Childress AR et al (2006). Cocaine cues and dopamine in dorsal striatum: mechanism of craving in cocaine addiction. I Neurosci 26: 6583-6588.

Wang GJ, Volkow ND, Telang F, Jayne M, Ma J, Rao M et al (2004). Exposure to appetitive food stimuli markedly activates the human brain. Neuroimage 21: 1790-1797.

Wang Z, Aguirre GK, Rao H, Wang J, Fernández-Seara MA, Childress AR et al (2008). Empirical optimization of ASL data analysis using an ASL data processing toolbox: ASLtbx. Magn Reson Imaging 26: 261-269.

Wong DF, Kuwabara H, Schretlen DJ, Bonson KR, Zhou Y, Nandi $A$ et al (2006). Increased occupancy of dopamine receptors in human striatum during cue-elicited cocaine craving. Neuropsychopharmacology 31: 2716-2727.

Wright P, He G, Shapira NA, Goodman WK, Liu Y (2004). Disgust and the insula: fMRI responses to pictures of mutilation and contamination. Neuroreport 15: 2347-2351.

Yacubian J, Sommer T, Schroeder K, Gläscher J, Kalisch R, Leuenberger B et al (2007). Gene-gene interaction associated with neural reward sensitivity. Proc Natl Acad Sci USA 104: 8125-8130.

Zahniser NR, Sorkin A (2004). Rapid regulation of the dopamine transporter: role in stimulant addiction? Neuropharmacology 47(Suppl 1): 80-91. 